\title{
FERROFLUID/ACTIVATED CARBON COMPOSITES FOR WATER PURIFICATION AND EMI SHIELDING APPLICATIONS
}

\author{
S.K. Barala ${ }^{1,2}$, M. Arora ${ }^{1}$, Chandni Puri ${ }^{1}$, \\ K.K.Saini ${ }^{1}$, R.K.Kotnala ${ }^{1}$, P.K.Saini ${ }^{1 *}$ \\ 1 CSIR-National Physical Laboratory, New Delhi-110012, India \\ ${ }^{2}$ Centre for Converging Technologies, University of Rajasthan, Jaipur-302004, India \\ *e-Mail: pksaini@nplindia.org
}

Activated carbon (AC) supported $\mathrm{Fe}_{3} \mathrm{O}_{4}$ nanoparticle-based composites (CFFs) have been prepared by mixing $\mathrm{AC}$ with a $\mathrm{Fe}_{3} \mathrm{O}_{4}$ ferrofluid followed by drying. These composites display a unique combination of electrical and magnetic properties along with nanoporosity. The structural, morphological and magnetic properties of the synthesized CFF composites have been characterized by XRD, SEM, TEM, VSM and TGA. The water purification capability has been demonstrated using methyl orange (MO) dye as an impurity. The porosity and magnetic properties enable fast adsorption of MO and magnetic separation of $\mathrm{MO}$ adsorbed $\mathrm{CFF}$ particles, respectively. In addition to water purification, these multifunctional CFFs may also find promising applications in catalyst separation, bioreactors, and for shielding electromagnetic interference in the microwave frequency range.

1. Introduction. Recently, nanoparticles have drawn enormous scientific attention due to their large surface to the volume ratio if compared to bulk materials and size-dependent properties resulting in technological applications [1]. Particularly, nanoparticulate magnetic materials like ferrites display unique properties, such as spin canting, surface anisotropy, superparamagnetic (SPM) response, etc. Ferrofluids (FFs) which represent stable dispersions of magnetic nanoparticles in a suitable liquid carrier have been directly employed in various applications [2]. These are also used in the development of multifunctional materials through the treatment under soft conditions of porous solids with FFs at a moderate temperature, which induces SPM properties in the final product and encourages their use in many advanced applications viz. water purification, magnetic catalytic support or enzymatic bioreactors, production of electro- or magneto-rheological fluids and for suppression of electromagnetic interference (EMI) [2-3]. We know that activated carbon (AC) is widely used in water filtration systems for purification purposes both at domestic front and in industry. As the density of this powder is very small, it becomes very difficult to handle and remove it from the purified water. In this consideration, the dry FF supported porous solids can solve the purification and separation problems. Further, the explosive growth of electronics has produced EMI as a side product [4]. To counter EMI, many materials with finite conductivity and dielectric/magnetic properties have been tried in the past [5-9]. Herein, we have prepared a multifunctional composite $(\mathrm{CFF})$ for simultaneous sorting out both of the above-mentioned problems, by attaching magnetic $\mathrm{Fe}_{3} \mathrm{O}_{4}$ particles with porous and electrically $\mathrm{AC}$ phase using a kerosene-based magnetite $\mathrm{FF}$ as a $\mathrm{Fe}_{3} \mathrm{O}_{4}$ source. This provides a means to optimize the porosity and electrical/magnetic properties of these composites to achieve maximum adsorption of impurities along with good EMI shielding properties. The structural, morphological and magnetic 
(a)

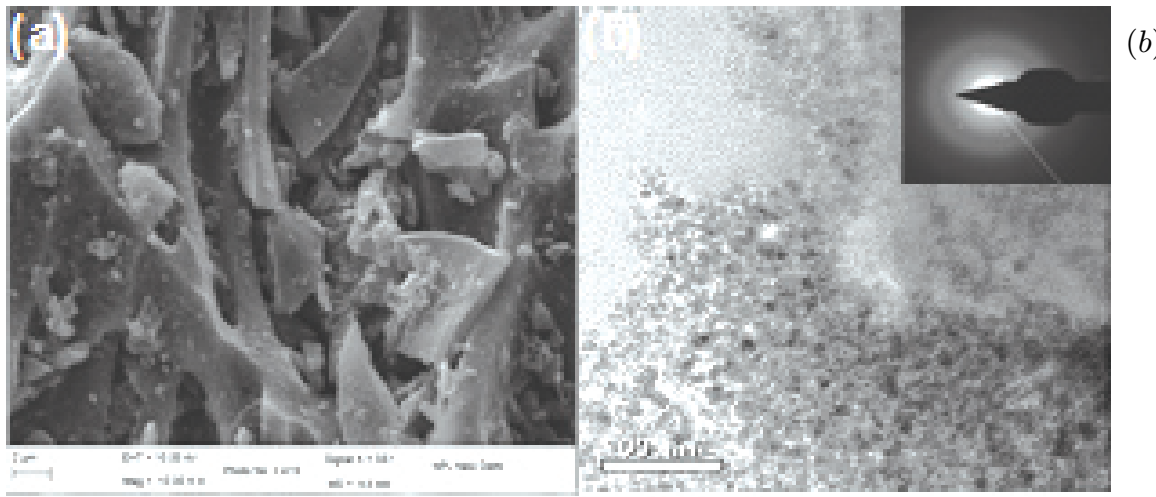

Fig. 1. (a) SEM, (b) TEM images of CFF and electron diffraction pattern (inset).

properties of these synthesized CFF samples were investigated by SEM, TEM, XRD, VSM and TGA techniques. This composite (CFF) has been demonstrated for purification of water having methyl orange (MO) dye as a model impurity. In addition, the EMI shielding capability of these composites has also been discussed.

\section{Experimental.}

2.1. Synthesis technique. A non-aqueous ferrofluid (FF) containing dispersion of oleic acid capped $\mathrm{Fe}_{3} \mathrm{O}_{4}$ nanoparticles in kerosene was used to produce an $\mathrm{AC}$ based composite $(\mathrm{CFF})$. In a typical procedure, the $\mathrm{FF}$ and $\mathrm{AC}$ were physically mixed to obtain a uniform and stable dispersion that was dried to remove the kerosene, leaving behind dry $\mathrm{Fe}_{3} \mathrm{O}_{4}$ particles over $\mathrm{AC}$ particles.

2.2. Structural characterization. XRD patterns were recorded on a Bruker diffractometer using $\mathrm{CuK} \alpha(\lambda=1.54059 \mathrm{~nm})$ as an X-ray source. The shape, size and distribution of these composites were defined with the help of SEM (ModelLEO 440) and TEM (Tecnai G2 F30 S-Twin). The magnetic properties of these samples were analyzed with Lakeshore make vibration (VSM). The thermal behaviour was studied by TGA (Mettler Toledo).

The SEM micrograph of the CFF composite (Fig. 1a) shows the presence of $\mathrm{Fe}_{3} \mathrm{O}_{4}$ clusters everywhere over the surface of porous AC particles. The porosity and magnetic character provide adsorption and magnetic actuation properties to the composite, respectively. Fig. $1 b$ shows the TEM image along with the corresponding electron diffraction pattern (inset). It is seen that the particles are almost spherical in shape with their average diameter in the range of $8-12 \mathrm{~nm}$. Further, the radial distance of diffraction spots gives an inter-planar spacing of $0.253 \mathrm{~nm}$ that matches with the planes of $\mathrm{Fe}_{3} \mathrm{O}_{4}[7]$.

Fig. 2 shows the powder X-ray diffraction patterns of AC, dry FF particles and their composite $(\mathrm{CFF})$. It can be seen that the $\mathrm{AC}$ is amorphous in nature and gives two broad peaks of $\sim 26^{\circ}$ and $\sim 44^{\circ}$ at the $2 \theta$ value. By contrast, the CFF shows an additional pronounced signal centered at $2 \theta \sim 35^{\circ}$, which dominates the peaks of the AC phase. This prominent peak matches well with the single crystalline phase of inverse cubic spinel structure of $\mathrm{Fe}_{3} \mathrm{O}_{4}$. The broadness of the $\mathrm{Fe}_{3} \mathrm{O}_{4}$ peak indicates the small crystallite size that was calculated from the Scherrer formula [9], and the average crystallite size varies between $8-10 \mathrm{~nm}$, which is in agreement with the TEM results.

The TGA plot of CCF (not shown) displays a three-step degradation: (i) small loss $\sim 100^{\circ} \mathrm{C}$ due to adsorbed moisture, (ii) loss of $\sim 13 \%$ in the $200-450^{\circ} \mathrm{C}$ range due to decomposition of oleic acid capping over $\mathrm{Fe}_{3} \mathrm{O}_{4}$ particles, and (iii) loss of $45 \%$ in the range $450-600^{\circ} \mathrm{C}$ due to the oxidation and decomposition of $\mathrm{AC}$. 


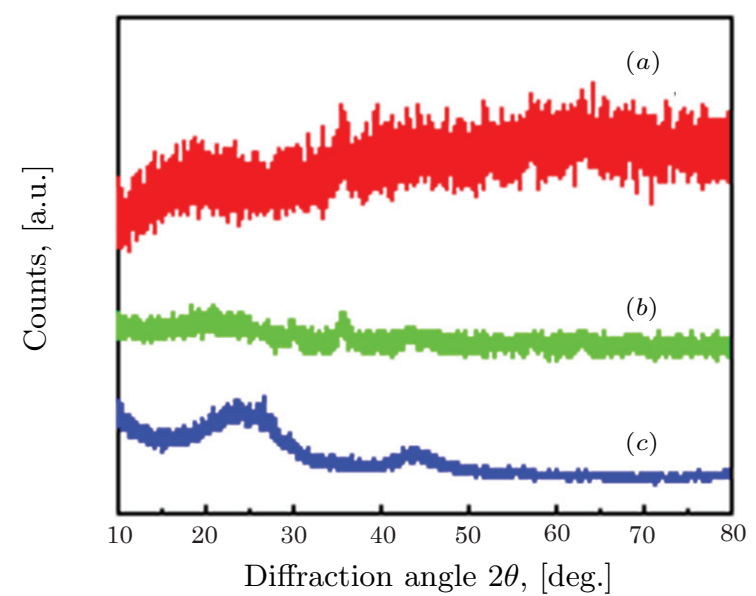

Fig. 2. XRD patterns of (a) dry ferrofluid (FF), (b) $\mathrm{AC} / \mathrm{FF}$ composite $(\mathrm{CFF})$ and $(c)$ of pure AC.

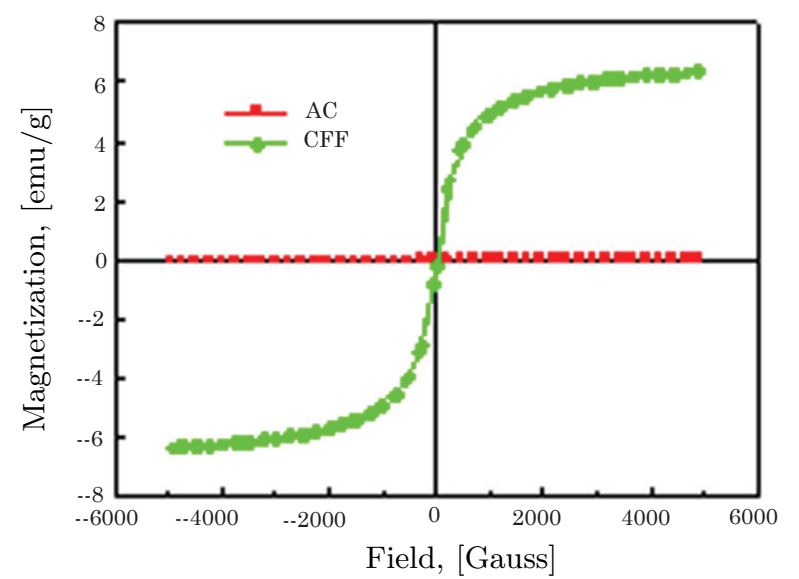

Fig. 3. VSM magnetization curve for the pure AC and CFF composite.

Nevertheless, the stability of the $\mathrm{CFF}$ up to $150^{\circ} \mathrm{C}$ indicates that they may be used and regenerated by heating up to $120^{\circ} \mathrm{C}$.

It can be seen that the AC exhibits a non-magnetic character (Fig. 3), whereas the CFF displays a typical super paramagnetic (SPM) behaviour with zero remanence and coercivity. The SPM of this sample originates from the fine crystallite size of $\mathrm{Fe}_{3} \mathrm{O}_{4}$ particles, which makes it easier for them to be thermally activated to overcome the magnetic anisotropy. The CFF shows a moderate saturation magnetization of $\sim 6.4 \mathrm{emu} / \mathrm{g}$, which may be useful for the magnetic separation of CFF particles once the absorption of pollutant is completed from the dirty water.

The water purification ability of CFF composites has been evaluated by using methyl orange (MO) dye in water as a pollutant. Fig. 4 shows a picture of the water having MO dye as an added impurity (left image). When CCF (composite) powder was added to this solution and mixture was agitated, the MO particles were selectively absorbed by the pores inside the CCF. These CCF particles with the adsorbed MO phase can be easily removed from the purified water (right image) via magnetic separation by placing a magnet at the bottom of the flask. It can be clearly seen that the water becomes completely transparent after treatment with the CCF indicating high separation efficiency. The calculations show that these 

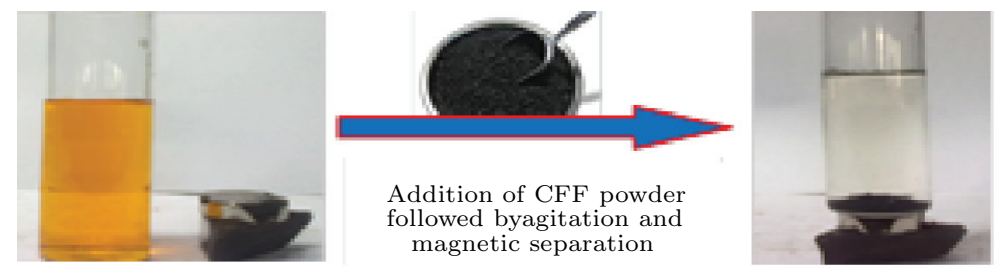

Fig. 4. MO solution (left image) and purified water (right image) with the magnetically pulled MO adsorbed CFF phase at the bottom of the flask (as a black powder).

composites exhibit a separation capacity of $\sim 9 \times 10^{-6} \mathrm{~mol}$ MO dye per gm of CCF powder. The shielding effectiveness (SE) can be expressed as [4-7], [10-13]:

$$
\mathrm{SE}(\mathrm{d} B)=10 \lg \frac{P_{\mathrm{t}}}{P_{\mathrm{i}}}=20 \lg \frac{E_{\mathrm{t}}}{E_{\mathrm{i}}}=-10 \log _{10}\left(\frac{\sigma_{\mathrm{T}}}{16 \omega \varepsilon_{0} \mu^{\prime}}\right)-8.68 t \sqrt{\frac{\sigma_{\mathrm{T}} \omega \mu^{\prime}}{2}},
$$

where $P_{\mathrm{i}}\left(E_{\mathrm{i}}\right)$ and $P_{\mathrm{t}}\left(E_{\mathrm{t}}\right)$ are the power (electric field strength) of incident and transmitted EM waves, respectively, whereas the parameters $t, \omega, \sigma_{\mathrm{T}}$ and $\mu^{\prime}$ are the shielding thickness, angular frequency, total conductivity and real permeability, respectively. Since the CFF composites have as conducting as magnetic properties, they are also expected to have promising applications in EMI shielding $[5,7]$ due to input impedance matching and shallow skin depth. The dielectric and magnetic properties of such multifunctional composites are governed by the $\mathrm{AC}$ and $\mathrm{Fe}_{3} \mathrm{O}_{4}$ phase, respectively [7]. Their detailed EMI studies are still under progress.

3. Conclusion. $\mathrm{Fe}_{3} \mathrm{O}_{4}$ loaded $\mathrm{AC}$ based composites materials with a combination of porosity and magnetic characteristics have been successfully prepared. These composites demonstrate a good water purification ability with sorption of $9 \times 10^{-6} \mathrm{~mol}$ MO dye per gram of CFF. Further, with coupled conducting and magnetic properties, these are also expected to have promising applications in EMI shielding in the microwave frequency range.

Acknowledgements. The authors are grateful to the Director of the CSIRNational Physical laboratory, Delhi, for providing the necessary research facilities.

\section{REFERENCES}

[1] Y.G. Alfaro, P. Aranda, F.M. Fernandes, B. Wicklein, M. Darder AND E.R. HitzKY. Adv. Mater., vol. 23 (2011), pp. 5224-5228.

[2] K. Safarik, K. Horska, K. Pospiskova and M. Safarikova. International Review of Chemical Engineering, vol. 4 (2012), pp. 346-352.

[3] S. Wang, Z.H. Zhu, A. Coomes, F. Haghseresht and G.Q. Lu. J. Colloids and Interface Sci., vol. 284 (2005), pp. 440-446.

[4] P. Saini, V. Choudhary, B.P. Singh, R.B. Mathur and S.K. Dhawan. Materials Chemistry and Physics, vol. 113 (2009), pp. 919-926, DOI: 10.1016/j.matchemphys.2008.08.065.

[5] P. Saini And M. Arora. New Polymers for Special Applications by Gomes AD, ed. (Intech, Croatia, 2012), DOI: 10.5772/48779 http://www.intechopen.com/download/pdf/38964.

[6] D.D.L. Chung. Carbon, vol. 39 (2001), pp. 279-285. 
Ferrofluid/activated carbon composites for water purification ...

[7] P. Saini, V. Choudhary, N. Vijayan and R.K. Kotnal. J. Phys. Chem. $C$, vol. 116 (2012), pp. 13403-13412. DOI: 10.1021/jp302131w.

[8] P. Saini and M. Arora. J. Mater. Chem. A, vol. 1 (2013), pp. 8926-8934, DOI: $10.1039 /$ C3TA11086A.

[9] P. Saini, V. Choudhary, K.N. Sood and S.K. Dhawan. J. Appl. Polym. Sci., vol. 113 (2009), pp. 3146-3155, DOI: 10.1002/app.30183.

[10] P. Saini and V. Choudhary. J. Nanoparticle Research, vol. 15 (2013), pp. 1-7, DOI: 10.1007/s11051-012-1415-2.

[11] P. Saini, M. Arora, G. Gupta, B.K. Gupta, V.N. Singh AND V. Choudhary. Nanoscale, vol. 5 (2013), pp. 4330-4336, DOI:10.1039/C3NR00634D.

[12] P. Saini And V. Choudhary. J. Mater Sci., vol. 48 (2) (2013), pp. 797-804, DOI: $10.1007 / \mathrm{s} 10853-012-6797-0$.

[13] R. Kumar, S.R. Dhakate, P. Saini, R.B. Mathur. RSC Advances, vol. 5 (2013), pp. 4145-4151, DOI: 10.1039/c3ra00121k.

Received 25.03.2013 